\title{
A reconfigurable leg for walking robots
}

\section{Journal Article}

Author(s):

Nan, Fang; Kolvenbach, Hendrik (D); Hutter, Marco (D)

Publication date:

2022-04

Permanent link:

https://doi.org/10.3929/ethz-b-000522681

Rights / license:

In Copyright - Non-Commercial Use Permitted

Originally published in:

IEEE Robotics and Automation Letters 7(2), https://doi.org/10.1109/LRA.2021.3139379 


\title{
A reconfigurable leg for walking robots
}

\author{
Fang Nan, Hendrik Kolvenbach, and Marco Hutter
}

\begin{abstract}
We present the design of a robotic leg that can seamlessly switch between a spring-suspended-, and unsuspended configuration. Switching is realized by a mechanism that exploits the alternative configuration of the two-link leg. The mechanism is lightweight, does not require additional actuation, and only relies on the leg movement for engagement. We validated the performance of the prototype leg on a single-leg testbed and investigated the power consumption during standing, crouching, and hopping in both configurations. The experiments showed that the efficiency of hopping and cyclic base height control is better with the spring-suspended configuration. However, it can undermine the leg's performance in position control and requires higher torque to maintain low base height, where the unsuspended configuration has advantages. Overall, the switching ability allows for seamlessly selecting the optimal mode for a specific locomotion task.
\end{abstract}

Index Terms-Legged Robots, Mechanism Design, Compliant Joints and Mechanisms

\section{INTRODUCTION}

D YNAMIC walking robots are often considered energetically inefficient compared to wheeled systems due to the need to actively compensate for the weight of the robot's main body. Many dynamic quadrupeds [1]-[4] are using torquedense motors with transparent transmissions to allow for high impact-robustness and torque-controllability, which, in turn, requires a constant draw of power to suspend the weight of the robot.

A method to decrease the torque demand of motors is using high gear reductions, leading to an increase in the friction losses of the system as well as the reflected inertia. This undermines the performance in high-speed force control, reduces the torque fidelity, efficiency, and the ability to mitigate hard impacts [5].

Energy losses become especially evident in situations where the robot has to stand for an extensive amount of time. Prime examples include the operation of wheeled-legged hybrids, where the robot is mostly rolling with intermittent walking phases to overcome obstacles. In this example, the weight of the torso still has to be actively compensated by the actuators during rolling, ultimately reducing the efficiency of the system [6]. Another example is found in planetary exploration, where robots traverse for relatively small distances, with frequent stops for scientific investigation or communication [7].

Manuscript received September 09, 2021; Revised November, 24, 2020; accepted December 15, 2020.

This paper was recommended for publication by Editor Tamim Asfour upon evaluation of the Associate Editor and Reviewers' comments.

This work has been conducted as part of ANYmal Research, a community to advance legged robotics. This work was supported in part by the Swiss National Science Foundation through the National Centres of Competence in Research Robotics (NCCR Robotics).

All the authors are with the Robotic Systems Lab (RSL) at ETH Zurich, Switzerland.

Correspondence should be addressed to Hendrik Kolvenbach (hendrikk@ethz.ch)

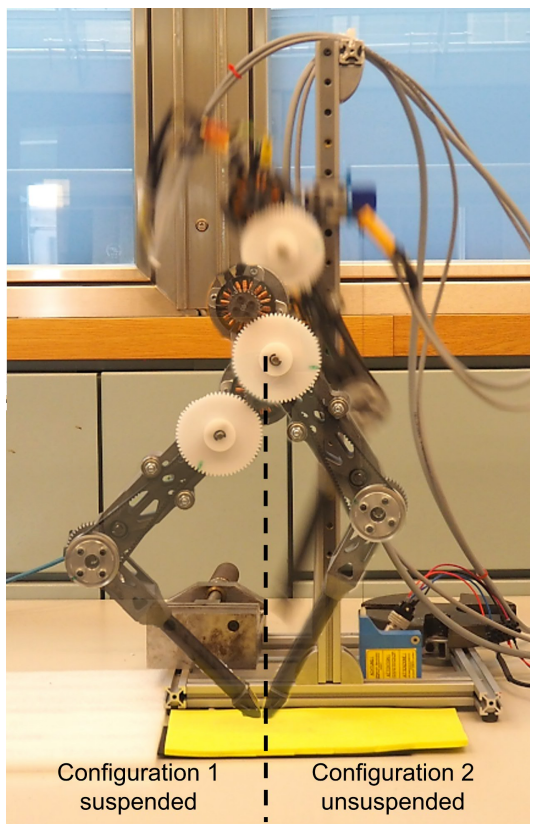

Fig. 1: Overlay picture of the spring-suspended and unsuspended configuration of the reconfigurable leg during jumping on the single-leg testbed.

In such a scenario, a legged robot is consuming precious energy for passive standing [8]. Previous research has shown that elasticity elements could be introduced to help support the robot's weight. Verstraten et al. [9] have shown that both parallel and series elastic actuators could have a torque reduction effect. Elastic elements have been used in multiple existing designs of robot legs including Salto-1P [10], Cheetah-Cub [11], and SpaceBok [12]. However, a downside of omnipresent (parallel) springs is the need for force compensation whenever a large range of motion is required. For example, the spring might help significantly for a defined locomotion task such as repetitive hopping but can be problematic when complex whole-body motions are required, such as taking samples or avoiding obstacles. While actively working against the spring is one aspect, a precise measurement or modeling of the spring force is necessary to avoid overshooting and enable precise force tracking of the feet, which introduces complexity to the system.

In recent years, a few designs of reconfigurable robot leg structures have been developed. For example, a compact electric clutch mechanism that controls the engagement of a parallel spring is developed in [13], leading to reduced energy consumption in different tasks. The design proposed in [14] can switch between three different configurations and has a significant advantage against traditional methods. 
However, these designs require additional actuators to trigger the switch, which adds mass and complexity to the system. Liu et al. [15] propose a passive switching design that automatically engages a parallel elasticity whenever the foot has contact to the ground and releases during the flying phase. While the leg showed excellent efficiency in hopping and leaping, this design may not perform efficient stance phase pose control due to the spring suspension.

This paper proposes a novel, lightweight design that exploits the so-far unused region in the joint space to add reconfigurability to robot legs without additional actuation. Our design uses a switch mechanism to engage a parallel elastic element such that the dynamics of the leg can be altered in the other half of the joint space. We designed and manufactured a fully actuated prototype and performed validation tests on a singleleg testbed. Our experiments show that the reconfigurable design has advantages compared to traditional leg designs. The unsuspended configuration is advantageous in position control tasks, while the suspension performs better during a hopping motion. The method enables the leg to seamlessly switch the configuration and reduce energy consumption depending on the desired locomotion task..

The following section introduces the multivalued nature of robot leg inverse kinematics and the principles on how robot leg design could take advantage of this. Section III introduces the design of our prototype, the selection of parameters, and the controllers. Section IV shows the result of a series of experiments performed on the prototype, followed by a brief discussion $\mathrm{V}$ and our conclusion VI.

\section{BACKGROUND}

The design of legs for robots is traditionally inspired by legs found in the animal kingdom [11], and thus, most of the existing designs have similar structures. The majority of dynamically walking quadrupeds have three Degrees of Freedom (DOF) legs that consist of two links, namely a thigh link and a shank link, connected in serial and driven by three independent actuators. The actuators describe the hip abduction/adduction (HAA), hip flexion/extension (HFE) and knee flexion/extension (KFE). The HFE and KFE joints allow the foot to have a large reach in the vertical plane.

Despite the apparent similarity between robot legs and animal legs, there is a fundamental difference between the sizes of their joint spaces. For most animals, the knee joint has a limited range of motion (smaller than $180^{\circ}$ [16]) due to the musclebone structure, while the motor-drive actuators used on robot legs can make use of complete rotations (e.g., ANYmal [17]).

The full rotation capabilities of some legged robots result in inverse kinematics with multiple solutions, i.e., there exist two solutions for joint positions given a desired foot position in the reachable zone. These solutions correspond to symmetrical points in the joint space. As a result, legged robots with full rotation capability commonly use only half of the available joint space to perform the tasks found in animals. Joint space data recorded with ANYmal during a demanding hike in the swiss alps* shows that, overall, far less than $10 \%$ of the available joint

*https://youtu.be/oPNkeoGMvAE

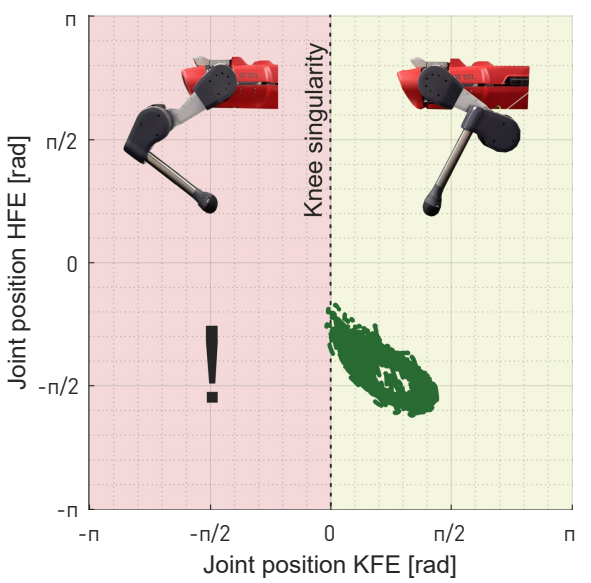

Fig. 2: Joint positions of KFE and HFE for the front left leg (in green) recorded during a hike with ANYmal compared to the theoretically available joint space.

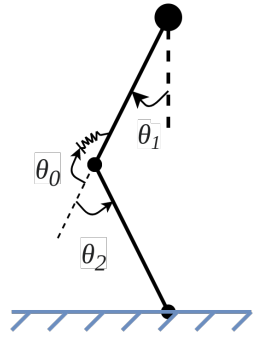

(a)

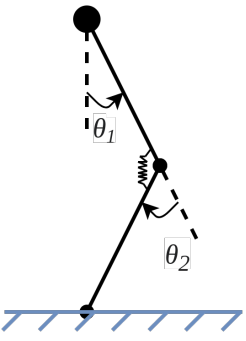

(b)
Fig. 3: Schematic view of the leg kinematics. The leg is able to operate in two different configurations, controlled by the angle of the KFE joint. In a) $\theta_{2}<0$ and the spring is not engaged. In b) $\theta_{2}>\theta_{0}>0$ and the spring is engaged.

space of HFE and KFE is used for locomotion. In particular, the robot is moving in an X-configuration, with both knees pointing inwards. By crossing the knee singularity, the robot has the option to move in O-configuration (Knees pointed outwards) and exploit the so far unused areas in the joint space (Figure 2). Therefore, with a properly designed switch mechanism, the robot leg has access to the two half-spaces of the configuration space, enabling different dynamics over the two halves without the need for additional actuators.

Our design takes advantage of this observation and uses a simple clutch mechanism to engage a parallel spring in one of the configurations. The concept of the design is shown in Figure 3. $\theta_{0}$ in the figure represents the spring engagement point, and $\theta_{1}$ and $\theta_{2}$ represent the position of the HFE and KFE joints, respectively. The engagement of the parallel spring is controlled purely based on the position of the KFE joint. It is essential to notice that the spring engagement point and the spring stiffness are the two parameters that define the nominal stance position and the dynamics of the suspended configuration.

Engaging the parallel spring allows the leg to stand passively in one configuration, with the spring supporting the system's weight even with a payload on the base. 


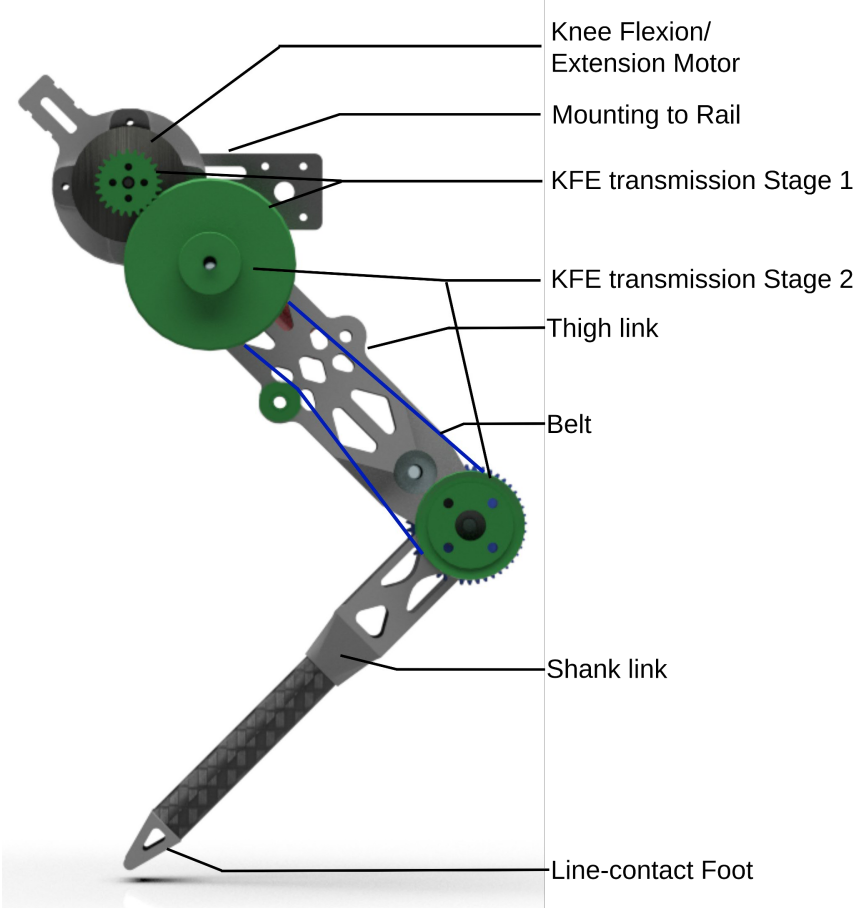

Fig. 4: Overview of the reconfigurable leg.

This function is also found in nature, where Flamingos use a specific joint posture and a locking mechanism that supports their body weight without any muscle activity [18]. So far, such a technique is not used in existing robot designs because the actuator has to compensate for the high spring torque in acyclic movements. Since the spring is only engaged in one of the two configurations in a reconfigurable design, this disadvantage can be avoided.

Compared to previous methods [13]-[15], our design is exceptionally lightweight and does not use any additional actuators to control the engagement of the elasticity since it is controlled passively by the angle of the leg's KFE joint position. While engaging a spring is a valuable feature in legged robotics, the unused configuration might also be used to enable other functionalities, i.e., the deployment of sensors.

\section{METHOD}

\section{A. Design Overview}

We developed a fully actuated leg prototype to verify the feasibility of our concept for a reconfigurable leg. An overview of the design is shown in Figure 4 and the specifications are listed in Table I.

For fast and convenient prototyping and testing, we selected the size of the leg to be $370 \mathrm{~mm}$ when fully extended and limited the total weight to less than $1 \mathrm{~kg}$. Similar values are found in other small-scale quadruped prototypes [3], [19]. Parts were printed out of Nylon PA12 or manufactured out of carbon fiber and aluminum to reduce the mass.

The prototype consists of an HFE and KFE joint, and the lengths of the thigh and shank links were selected to be the

\begin{tabular}{ll}
\hline Specification & Value \\
\hline Fully extended length & $370 \mathrm{~mm}$ \\
Total mass & $0.83 \mathrm{~kg}$ \\
KFE spring constant (designed/measured) & $(1.23 / 1.0) \mathrm{N} \mathrm{m} \mathrm{rad}^{-1}$ \\
Rotational spring engagement angle & $30^{\circ}$ \\
Maximum hip joint actuator torque & $1.20 \mathrm{~N} \mathrm{~m}$ \\
Maximum knee joint actuator torque & $3.14 \mathrm{~N} \mathrm{~m}$ \\
Base height in suspended stance & $348 \mathrm{~mm}$ \\
\hline \hline
\end{tabular}

TABLE I: Specification of the leg prototype,

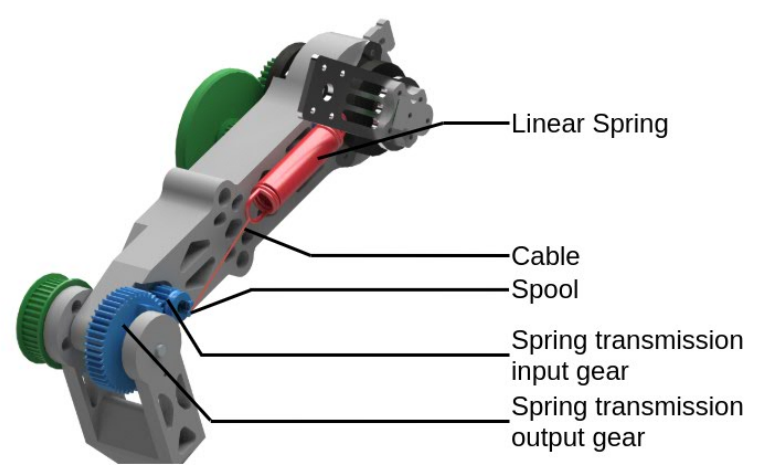

Fig. 5: Detailed view of the spring and clutch mechanism.

same to maximize the reachability. The HFE joint is actuated with a T-Motor 5008 motor $^{\dagger}$, and the KFE joint is actuated with a T-Motor 4006 motor ${ }^{\ddagger}$. Both actuators were placed at the hip, which reduces the weight and inertia of the links. Each motor is equipped with a 12-bit RLS RM08 magnetic encoder and Elmo Gold Twitter motor drive. The motor controllers are placed next to the leg and do not contribute to the overall mass. A laboratory power supply powers the leg.

The hip motor directly drives the HFE joint, as the hip joint requires a smaller torque in hopping tasks than the KFE joint. A two-stage transmission system (green components in Figure 4) with an overall gear ratio of 5.93 transfers the torque to the knee joint and increases the maximum torque at the joint to $3.14 \mathrm{~N} \mathrm{~m}$. In detail, the first stage of the transmission involves two spur gears with 18 and 48 teeth, respectively, amplifying the maximum torque to $1.42 \mathrm{Nm}$, which is 2.67 times higher than the maximal motor output.

The second stage, a belt transmission, amplifies the torque again by a factor of 2.22 , amounting to $3.14 \mathrm{~N} \mathrm{~m}$ peak torque, and delivering it to the knee location. The 3GT timing belt was selected for its ability to transmit high torque with a relatively compact size. The smaller pulley with 18 teeth is fixed on an intermediate shaft with the bigger gear, and the bigger pulley is directly fixed to the shank link at the knee joint. A ball bearing on the thigh link acts as an idler to provide pre-tensioning for the belt. The line-contact foot of the robot is made of 3D printed Nylon PA12 with a glued-on rubber sole for high traction.

\section{B. Switch mechanism}

On the opposing side of the thigh link, we installed the clutch mechanism and the spring (Figure 5). A linear spring with

\footnotetext{
${ }^{\dagger}$ https://uav-en.tmotor.com/html/2020/Antigravity_0422/340.html

‡https://uav-en.tmotor.com/html/2018/Antigravity_0402/24.html
} 


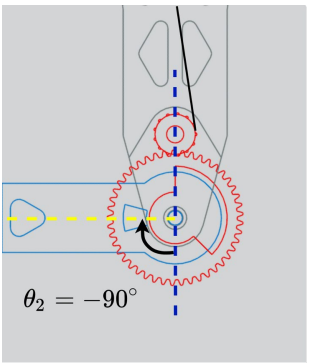

(a)

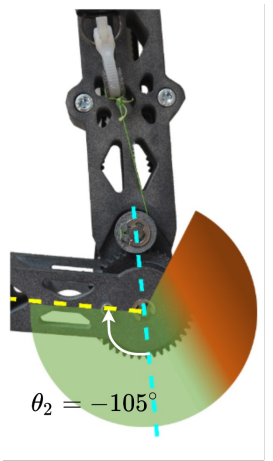

(d)

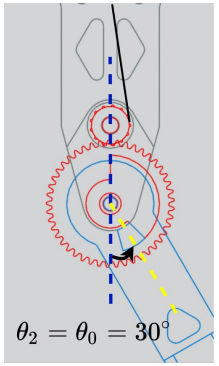

(b)

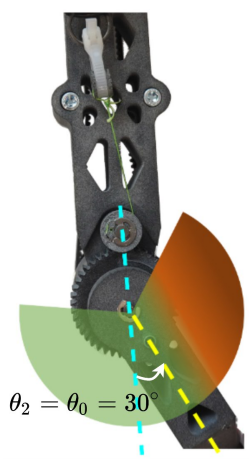

(e)

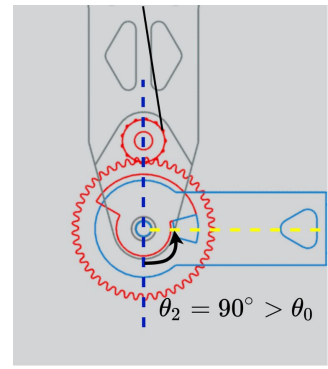

(c)

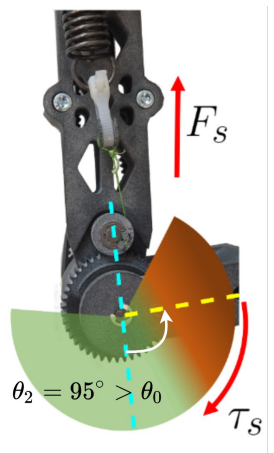

(f)
Fig. 6: Different states of the clutch mechanism. Pictures a), b), c) show the CAD design, and d), e), f) show the prototype. In a), b), and c) the blue parts are the shank link, the gray parts belong to the thigh link, and the red parts are the transmission for the spring. The red and the blue sectors are the contact surfaces that transmit the force between the spring and the shank link, and they are connected only when $\theta_{2} \geq \theta_{0}$. In a) and d) the contact surfaces are disconnected and the spring is not engaged. In b) and e) the two surfaces come into contact and the spring is engaged when the shank link continues to rotate in the counter-clockwise direction, as shown in c) and f).

$3.1 \mathrm{~N} \mathrm{~mm}^{-1}$ stiffness is used here such that it is conveniently adjustable. The details of the spring selection can be found in Sec. III-C. One side of the spring is fixed to the link, and the other side is tied via a zip tie to a braided fishing line made of non-stretchable Polyethylene. The zip tie is used to adjust the tension of the spring. The other terminal of the cable is winded on a spool. Thus, the linear force generated by the spring is shifted into torque on the spool. The spool then transfers the torque to the shank link through another set of spur gears. The diameter of the cable spool and the gear ratio can be adjusted so that different spring parameters can be obtained even with a limited selection of springs.

We designed the clutch mechanism to have the correct spring engagement behavior by connecting and disconnecting the gear to the shank link. We installed two sector-shaped structures on the shank link and the output gear of the spring transmission to form a one-direction transmission. The angles of the two sectors were carefully selected. When the leg operates in the joint space where $\theta_{2}<0$, the transmission is disconnected. Thus, the shank link can rotate independently of the gear,

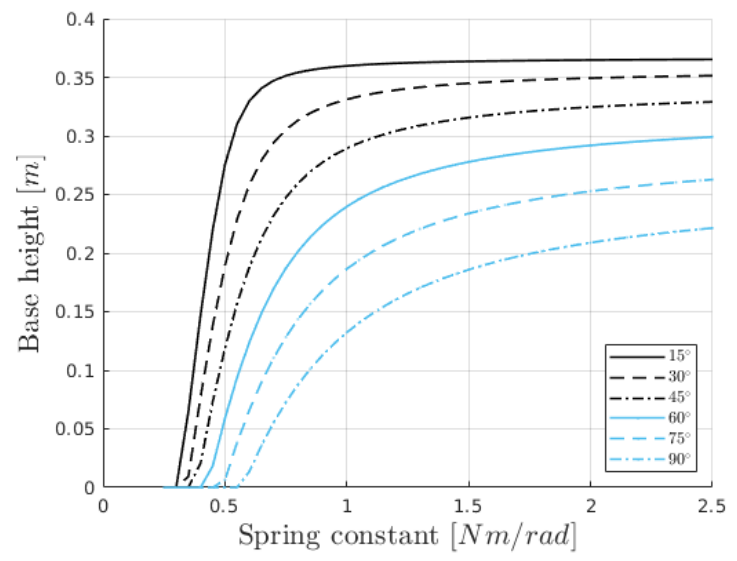

Fig. 7: Base height in passive stance depending on selected spring constant and spring engagement point.

maintaining the neutral position under the position limiting mechanism and the spring. This state is shown in Figure 6a. The gear does not rotate in this state, and the cable connected to the spring is only pre-tensioned. As the shank link rotates to the other half of the configuration space and reaches the spring engagement point, the piece on the shank link touches the sector on the gear and engages the spring, as shown in Figure $6 \mathrm{~b}$. As the shank link continues to rotate, the gear rotates with the link, and the two sectors transmit the torque generated by the spring to the shank link. This mode of operation is shown in Figure 6c. The design of the switch mechanism only involves a set of spur gears and the additional sector-shaped piece on the shank link. The total mass, including the spring, is merely $36.7 \mathrm{~g}$, less than $5 \%$ of the mass of the whole leg.

\section{Spring selection}

The important parameters for the parallel spring include the spring constant and the engagement point.

In order to allow the desired passive stance operation, there has to be an equilibrium where the spring torque balances the gravitational effects. Although the equilibrium could exist with many spring constant-engaging point combinations, the base height when the leg performs passive stance could be very different. Using the approximate mass properties obtained from the model, the calculations on the standing equilibrium have been performed for different spring constants and engagement points (Figure 7).

For the energy storage purpose of the parallel spring, it is desired to have higher storage capacity, which suggests a lower spring engagement angle and higher spring constant. However, to fully use the energy storage function, we would like the leg to reach a full crouch with the actuator, which suggests that the spring torque, when fully crouched, should not exceed the maximum torque of the KFE actuator. Considering all the requirements and the availability of off-the-shelf spring models, the spring constant was selected to be $1.23 \mathrm{~N} \mathrm{~m} \mathrm{rad}^{-1}$, which is achieved with a linear spring with a $3.09 \mathrm{~N} \mathrm{~mm}^{-1}$ spring constant, and appropriate transmission. 


\section{Controller}

To control the leg prototype, we mainly used controllers based on Inverse Dynamics (ID) and Virtual Model Control (VMC) [20]. With our two DOF robot leg and its base mounted on a vertical rail, we chose the base height $z_{B}$ and the HFE joint position $\theta_{1}$ as well as the KFE joint position $\theta_{2}$ as generalized coordinates, represented by

$$
\mathbf{q}=\left[\begin{array}{lll}
z_{B} & \theta_{1} & \theta_{2}
\end{array}\right]
$$

For the stance control, a simple PD controller on the base height was applied. The control law is described by the following equations:

$$
\begin{aligned}
& \ddot{z}_{B}^{*}=k_{p}\left(z_{B}^{*}-z_{B}\right)-k_{d} \dot{z}_{B}, \\
& \ddot{\mathbf{q}}^{*}=\mathbf{J}_{B}^{+}\left(\ddot{z}_{B}^{*}-\dot{\mathbf{J}}_{B} \dot{\mathbf{q}}\right), \\
& \boldsymbol{\tau}_{S}=\left(\mathbf{N}_{C} \mathbf{S}\right)^{+} \mathbf{N}_{C}^{T}\left(\mathbf{M}(\mathbf{q}) \ddot{\mathbf{q}}^{*}+\mathbf{b}(\mathbf{q}, \dot{\mathbf{q}})+\mathbf{g}(\mathbf{q})\right) .
\end{aligned}
$$

In (2), the desired base acceleration $\ddot{z}_{B}^{*}$ is calculated using base position reference, $z_{B}^{*}$, with a PD control law. $k_{p}$ and $k_{d}$ are the proportional and derivative gains, respectively. Then Eqn (3) calculates the desired acceleration in generalized coordinate directions, $\ddot{\mathbf{q}}^{*}$. The base Jacobian $\mathbf{J}_{B}$ is the matrix that maps the generalized velocity $\dot{\mathbf{q}}$ to base velocity, and the super script + represents the Moore-Penrose inverse. The required joint torque, $\tau_{S}$, is then computed from (4), the support-consistent dynamics of the leg, in which $\mathbf{N}_{C}$ describes the null space of the contact Jacobian, $\mathbf{S}$ the selection matrix for the actuated joints and $\mathbf{M}, \mathbf{b}$ and $\mathbf{g}$ the system inertia matrix, Coriolis and centrifugal terms as well as gravitational terms, respectively. For details on support-consistent dynamics, we refer the readers to [21].

The VMC controller is used during the stance phases when hopping [22]. When the contact between the foot and the ground is detected, the VMC emulates a virtual spring between the base of the leg base and the ground. This provides a simple and intuitive way that allows the leg to perform continuous hopping. The force acting on the base exerted by the virtual spring is mapped to joint torques. For certain spring stiffness $k_{p s}$ and damping $k_{d s}$, the virtual spring force acting on the base, $\mathbf{F}_{b}$ could be represented as

$$
\mathbf{F}_{b}=k_{p s}\left[\begin{array}{lll}
0 & 0 & z_{B}^{*}-z_{B}
\end{array}\right]^{T}-k_{d s}\left[\begin{array}{lll}
0 & 0 & \dot{z}_{B}
\end{array}\right]^{T} .
$$

Consider the contact-consistent dynamics of the leg

$$
\underline{\mathbf{M}}(\mathbf{q}) \ddot{\mathbf{q}}+\underline{\mathbf{b}}(\mathbf{q}, \dot{\mathbf{q}})+\mathbf{g}(\mathbf{q})=\mathbf{S}^{T} \boldsymbol{\tau}+\mathbf{J}_{c}^{T} \mathbf{F}_{c},
$$

in which the nonlinear dynamics are neglected. Using quasistatic approximation in stance, the contact force $\mathbf{F}_{c}$ can be approximated by the total gravity force of the system. Then the desired torque to emulate the virtual spring is given by

$$
\boldsymbol{\tau}_{V M C}=\mathbf{S}\left(-\mathbf{J}_{c, r}^{T} \mathbf{F}_{c}-\mathbf{J}_{B F, r}^{T} \mathbf{F}_{b}+\mathbf{g}\right),
$$

where $\mathbf{J}_{c, r}$ is the Jacobian that maps $\dot{\mathbf{q}}$ to the velocity of the foot in inertia frame, and $\mathbf{J}_{B F, r}$ is the Jacobian that maps $\dot{\mathbf{q}}$ to the velocity of the foot with respect to the base. During the hopping experiments, the controller switches to flight-phase control after obtaining enough base velocity to takeoff. The flight-phase controller uses a inverse dynamics control law to maintain the foot position with respect to the base, given by the following equations:

$$
\begin{aligned}
\ddot{\mathbf{q}}^{*} & =k_{p f}\left(\mathbf{q}^{*}-\mathbf{q}\right)-k_{d f} \dot{\mathbf{q}}, \\
\boldsymbol{\tau}_{I D} & =\mathbf{S}^{+}\left(\mathbf{M}(\mathbf{q}) \ddot{\mathbf{q}}^{*}+\mathbf{b}(\mathbf{q}, \dot{\mathbf{q}})+\mathbf{g}(\mathbf{q})\right) .
\end{aligned}
$$

where $\mathbf{q}^{*}$ is the desired joint position calculated with inverse kinematics, and $k_{p f}$ and $k_{d f}$ are the PD gains.

Before applying the torque command from any of the aforementioned controllers, the spring torque is compensated if necessary following

$$
\boldsymbol{\tau}_{a c t}=\left\{\begin{array}{cl}
\boldsymbol{\tau}^{*} & \theta_{2} \leq \theta_{0} \\
\boldsymbol{\tau}^{*}-\left[\begin{array}{c}
0 \\
k_{s}\left(\theta_{2}-\theta_{0}\right)
\end{array}\right] & \theta_{2}>\theta_{0}
\end{array},\right.
$$

with $k_{s}$ being the measured spring constant, and $\tau^{*}$ the desired torque given by the active controller. Switching control between the configurations is realized by a single jump of the robot.

\section{EXPERIMENTAL ANALYSIS}

\section{A. Experimental setup}

We mounted the leg to a rail which allows linear, vertical movement with minimum friction (Figure 1). We investigated three test cases: stance, crouching and hopping. Each of these tests was performed in suspended and unsuspended configurations to compare the results. As mentioned in III-B, the proposed switch mechanism and spring is lightweight. Therefore, we assume that the leg's performance in the unsuspended configuration can be approximated by its performance without the proposed reconfigurable design. During the experiments, we recorded the state of the leg, actuator torques as well as voltage and current readings from the power supply. We noticed that the actual spring parameters were different from the values in the original design as stated in Table I. The mismatch could be caused by the non-linearity of the spring or the elasticity of other elements in the system. For the experiments, we identified the spring model based on actuator data.

\section{B. Experimental results}

1) Stance experiments: For the leg's stance performance, the critical difference in the two configurations is the actuator torque on the knee joint caused by the engaged parallel elasticity. The knee joint torque measurements were taken when the leg prototype stood stably at different base height values. The measurements in both suspended and unsuspended configurations are plotted against the base height, as shown by the dashed lines in Figure 8.

The black dashed curve in the plot shows the torque required to support the leg in unsuspended stance mode at different base heights, while the red curve shows that in the suspended configuration. The torque values are positive in both configurations, meaning that the knee actuator is acting against the spring in the suspended mode.

In the stance task of robot legs, the joint positions remain constant. Hence, the energy consumption only comes from the thermal loss of the motors, which is related to the torque 


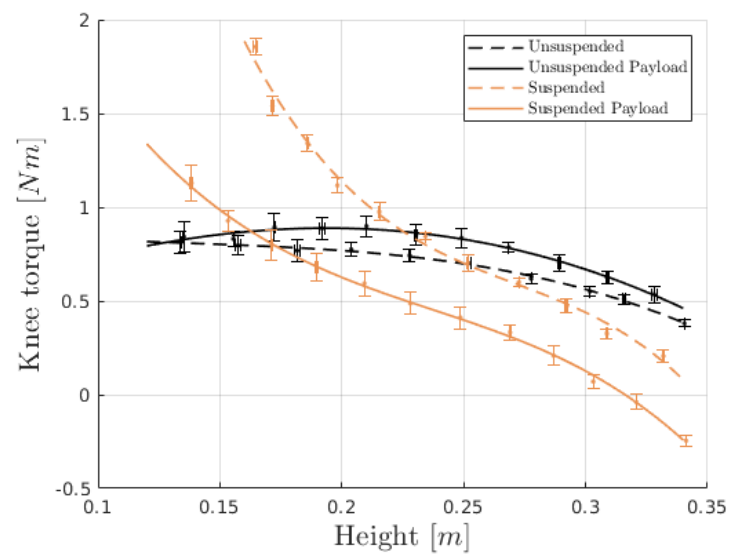

Fig. 8: Knee joint actuator torque during stance at different base heights.

generated by the motor. Therefore, the configuration that requires lower motor torque to stand is preferred to reduce the leg's energy consumption. The crossing point of the two dashed curves in Figure 8 provides the threshold to determine which configuration is more efficient for a required base height. According to the plot, the spring-suspended configuration is preferred when the desired base height is high, while the unsuspended configuration is more efficient at a lower stance height.

This experiment suggests that the unsuspended mode is favorable in most cases, as the crossing point of the curves is around $0.25 \mathrm{~m}$, more than $60 \%$ of the fully extended length of the leg. However, in most real-world applications of legged robots, the leg carries an additional payload, which requires additional torque on the knee joint to support itself. With a $0.25 \mathrm{~kg}$ additional payload, the experiment was repeated and the data is plotted with solid curves in Figure 8. the crossing point of the two curves is at about $0.16 \mathrm{~m}$. When the desired stance height is higher than this value, the KFE actuator torque is lower in the spring-suspended configuration as the spring is able to support the leg. This covers $78 \%$ the range of the leg's possible stance height, suggesting the suspended configuration is preferable.

Overall, we conclude from the experiments that the advantage in efficiency for extended stance of the leg depends on the payload situation and the desired height. In practice, one could select the proper spring stiffness and engagement point of the spring such that the weight of the robot can be supported passively by the spring in the spring-suspended configuration. Taking advantage of the reconfigurable design, in case the robot needs to stand with reduced height, the unsuspended configuration can always be used to save energy.

2) Crouching experiments: We performed a crouching test with the two configurations of the prototype, in which a cyclic base height reference with $0.3 \mathrm{~Hz}$ frequency is given, mimicking a stance-phase motion. The result is shown in Figure $9 \mathrm{a}$ and Figure $9 \mathrm{~b}$ respectively. According to the measurements taken on the power supply, the crouching motion in the springsuspended configuration consumes $115.02 \mathrm{~J}$ energy per crouch on average. In contrast, in the unsuspended configuration, the average energy consumption per crouch is $140.32 \mathrm{~J}$. The spring-suspended configuration is $18 \%$ more efficient mostly because of the reduced effort during the increase of the base height with the help of the spring. It was also observed that the peak torque required in the suspended configuration is $13 \%$ higher than the unsuspended one, which comes from the spring torque compensation when the leg is in the deep crouched state. In addition, the base height reached in the two configurations is also different despite the reference height and controller parameters being the same. The lowest crouch reached with the spring-suspended configuration is $8 \mathrm{~mm}$ higher compared to that in the unsuspended configuration. We believe this difference comes from the inaccuracy of the spring model. Although we have identified the spring parameters after building the prototype, the actual spring dynamics could be nonlinear and might change slightly during the experiments as certain delicate parts in the system might deform.

3) Hopping experiments: Next, we performed a hopping test mimicking a complete locomotion task. The parameters of the VMC controller were tuned such that stable and repetitive hopping to a height of $0.415 \mathrm{~m}$, which is $112 \%$ of the fully extended length of the leg, could be achieved with both configurations.

Figure 10a shows a section of the data in the hopping experiment with the unsuspended configuration, and Figure $10 \mathrm{~b}$ corresponds to that in the suspended configuration.

We integrated the power data for more than 60 repetitive hopping cycles and averaged the energy consumption per hop in the unsuspended configuration, which yielded $84.4 \mathrm{~J}$ In the suspended configuration, however, the average energy consumed was less at $49.3 \mathrm{~J}$. With the same hopping, the height achieved by the suspended configuration is $41.6 \%$ more efficient. It can be observed from the energy plots that the energy saving is caused mainly by the reduced power during the stance phases. In the continuous hopping experiments, every stance period consists of two different processes: a deceleration process, when the leg goes into a crouch and reduces the base speed to zero, and an acceleration process, when the leg accelerates again to perform the next hop. For the configuration without the parallel spring, the effort needed on the knee joint to decelerate and accelerate all comes from the actuator. For the suspended configuration, the parallel spring stores energy during the deceleration and releases energy during the acceleration. This reduces the energy consumption and significantly reduces the peak torque needed from the KFE actuator. Similar findings have been reported in [12] for a larger quadruped. In the plot, the average value of the peak KFE actuator torque in each cycle is $2.27 \mathrm{Nm}$ for the unsuspended configuration and $0.92 \mathrm{Nm}$ for the suspended configuration, yielding a reduction by $59.5 \%$.

\section{DISCUSSION}

\section{A. Mechanism reliability}

We did not encounter a single failure of the clutching mechanism during our experiments despite repetitive and extensive testing and tuning. However, due to the small scale 

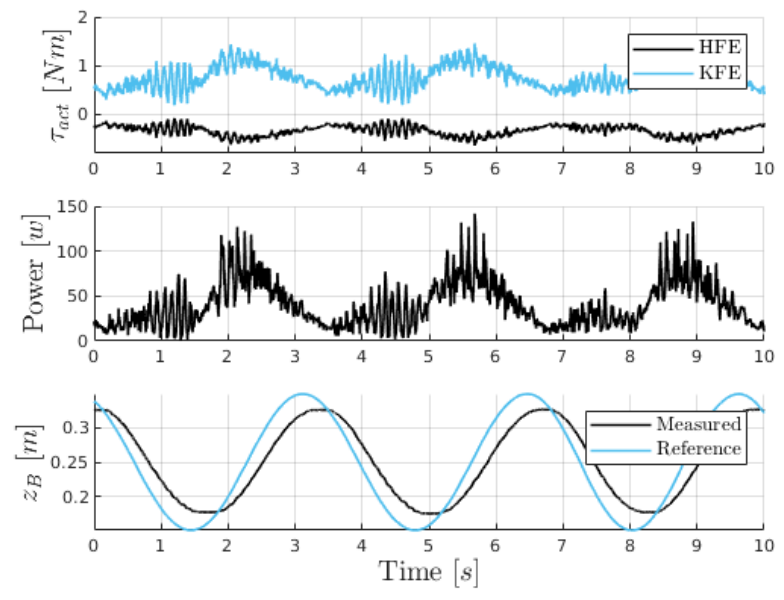

(a) Unsuspended configuration
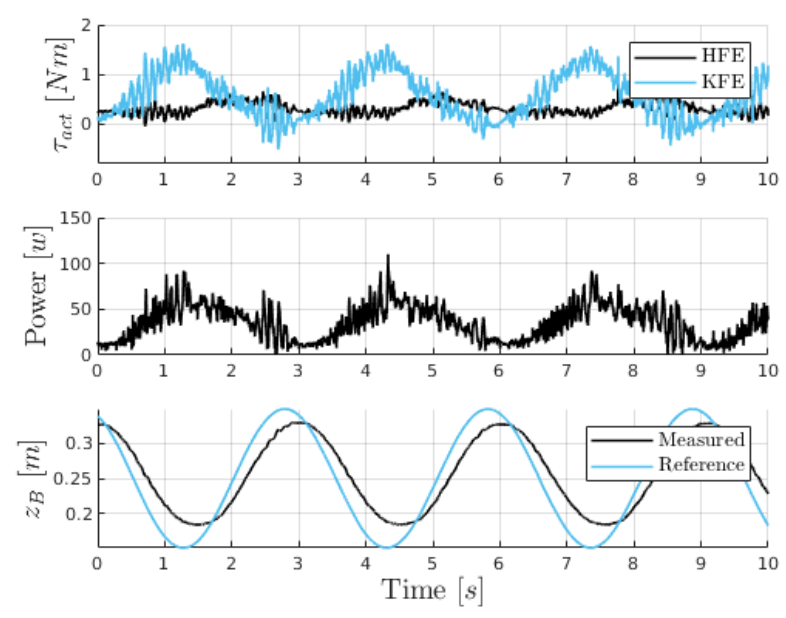

(b) Suspended configuration

Fig. 9: Actuator torque, power consumption and base height of the reconfigurable leg during the crouching experiments.
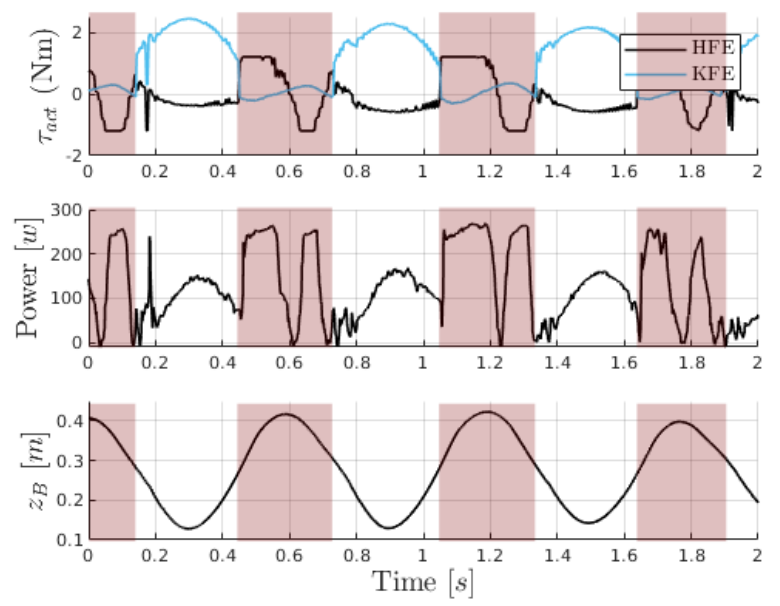

(a) Unsuspended configuration
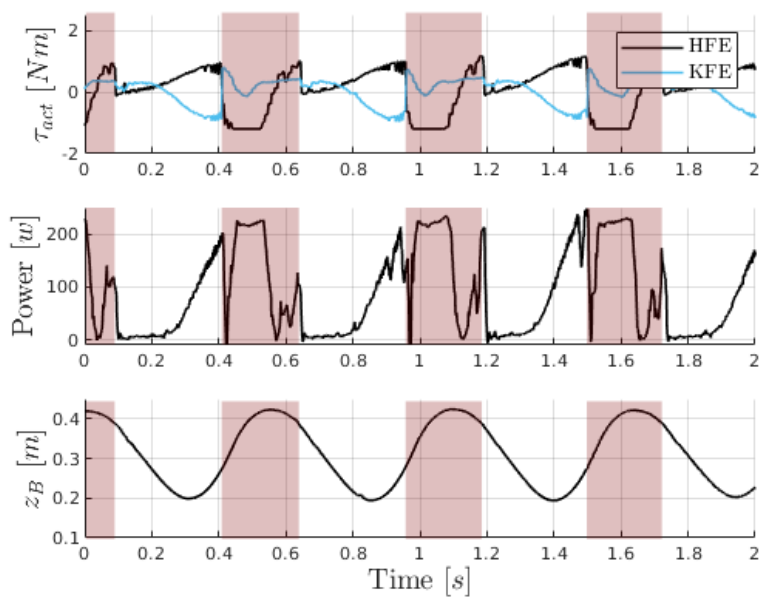

(b) Suspended configuration

Fig. 10: Actuator torque, power consumption and base height of the reconfigurable leg during the hopping experiments. The sections marked in red corresponds to flying phases.

of the prototype and the high spring forces, the installation of the spring is challenging and requires a cable material with sufficient strength. Means to optimally attach cables to mechanical structures, such as by splicing and braiding [22] might be considered in future iterations of the work.

\section{B. Spring parameter selection}

The experiments confirmed that the efficiency of the leg is highly affected by the spring. In cyclic base motion and hopping experiments, the spring suspension brought a significant advantage in terms of energy efficiency. During stance, the efficiency depends on the payload state and desired height.

The main parameters affecting the efficiency of each task are the spring engagement point and spring coefficient, which have to be carefully selected. For example, a high spring coefficient and early spring engagement point might be more beneficial to compensate a heavy payload passively. At the same time, it reduces the reachable base heights due to the torque saturation of the motors during crouch. During the flying phase, the actuator would also need to actively compress the spring to keep the joint position away from the singularity.

\section{Drawbacks caused by the spring}

A key advantage of the reconfigurable leg design is the ability to use a parallel elasticity on the leg's KFE joint that is strong enough to support the leg's weight. If not accurately estimated, a too high spring force undermines the control performance or even leads to unstable behaviors. We characterized the spring after mounting by measuring the joint torque data and found a discrepancy compared to the datasheet, which required tedious modeling in the assembled state. An alternative would be to 
install force sensors, similar to [12], at the cost of added mass and complexity.

In addition, compensating for the spring force reduces the joint torque available for control tasks, especially when the spring is extended. For example, when the leg operates in the spring-suspended configuration and the base-to-foot distance equals the link length, $62 \%$ of the total KFE joint torque is used to compensate for the spring. Therefore, it would be preferable to switch to the other configuration to avoid the limitation in control performance.

\section{CONCLUSION}

In conclusion, we present the design of a fully actuated reconfigurable leg prototype with two configurations, namely a spring-suspended configuration and an unsuspended one. Switching is realized by crossing the singularity of the leg. A passive clutch mechanism hereby engages or disengages the spring. We discovered that each configuration provides a benefit depending on tasks commonly used in legged robots.

We selected the spring parameters to allow for passive stance and provide high elastic energy storage capacity. Depending on the spring stiffness and engagement point, the spring can suspend the robot at certain base heights when standing passively. Experiments showed that hopping is more energy efficient with the engaged spring and allows for energy savings of up to $40 \%$. However, the peak actuator torque in one direction is drastically reduced due to torque compensation for the spring. The use of the parallel elasticity also requires additional effort to accurately model and compensate the spring torque. Thus, the unsuspended configuration could provide better performance in rapid and precise joint position control.

Overall, we conclude that the reconfigurable design takes advantage of the so-far unused areas of the joint space by providing additional functionality with little design overhead. The option to reconfigure a leg might be especially useful in application scenarios where robots have to switch frequently between locomotion and passive suspension tasks, such as those found when driving with wheeled-legged hybrids or performing planetary exploration.

A natural follow-up of this work is to investigate the advantage of the reconfigurable design in a full multi-legged robot. To perform such an evaluation, it is necessary to introduce a locomotion policy that is aware of the two leg modes and selects the appropriate configuration based on the environment and optimization criteria.

\section{ACKNOWLEDGEMENT}

We would like to thank Nico Kurth for his concept ideas on the switch mechanism, as well as Philip Arm and Jonas Junger for their help with the experimental setup.

\section{REFERENCES}

[1] D. J. Blackman, J. V. Nicholson, C. Ordonez, B. D. Miller, and J. E. Clark, "Gait development on Minitaur, a direct drive quadrupedal robot," in Unmanned Systems Technology XVIII, vol. 9837. SPIE, May 2016, pp. $141-155$.

[2] P. Arm, et al., "Spacebok: A dynamic legged robot for space exploration," in 2019 International Conference on Robotics and Automation (ICRA). IEEE, 2019, pp. 6288-6294.
[3] B. Katz, J. D. Carlo, and S. Kim, "Mini Cheetah: A Platform for Pushing the Limits of Dynamic Quadruped Control," in 2019 International Conference on Robotics and Automation (ICRA), May 2019, pp. 62956301.

[4] N. Kau, A. Schultz, N. Ferrante, and P. Slade, "Stanford doggo: An opensource, quasi-direct-drive quadruped," in 2019 International Conference on Robotics and Automation (ICRA), 2019, pp. 6309-6315.

[5] J. E. Pratt and B. T. Krupp, "Series Elastic Actuators for legged robots," in Unmanned Ground Vehicle Technology VI, vol. 5422. International Society for Optics and Photonics, Sept. 2004, pp. 135-144.

[6] M. Bjelonic, R. Grandia, O. Harley, C. Galliard, S. Zimmermann, and M. Hutter, "Whole-Body MPC and Online Gait Sequence Generation for Wheeled-Legged Robots," arXiv:2010.06322 [cs, eess], July 2021.

[7] A. R. Vasavada, et al., "Overview of the Mars Science Laboratory mission: Bradbury Landing to Yellowknife Bay and beyond," Journal of Geophysical Research: Planets, vol. 119, no. 6, pp. 1134-1161, 2014.

[8] H. Kolvenbach, et al., "Traversing Steep and Granular Martian Analog Slopes With a Dynamic Quadrupedal Robot," arXiv:2106.01974 [cs], June 2021

[9] T. Verstraten, P. Beckerle, R. Furnémont, G. Mathijssen, B. Vanderborght, and D. Lefeber, "Series and Parallel Elastic Actuation: Impact of natural dynamics on power and energy consumption," Mechanism and Machine Theory, vol. 102, pp. 232-246, Aug. 2016.

[10] D. W. Haldane, J. K. Yim, and R. S. Fearing, "Repetitive extremeacceleration (14-g) spatial jumping with Salto-1P," in 2017 IEEE/RSJ International Conference on Intelligent Robots and Systems (IROS), Sept. 2017, pp. 3345-3351.

[11] A. Spröwitz, A. Tuleu, M. Vespignani, M. Ajallooeian, E. Badri, and A. J. Ijspeert, "Towards dynamic trot gait locomotion: Design, control, and experiments with Cheetah-cub, a compliant quadruped robot," The International Journal of Robotics Research, vol. 32, no. 8, pp. 932-950, July 2013.

[12] H. Kolvenbach, E. Hampp, P. Barton, R. Zenkl, and M. Hutter, "Towards Jumping Locomotion for Quadruped Robots on the Moon," in 2019 IEEE/RSJ International Conference on Intelligent Robots and Systems (IROS), Nov. 2019, pp. 5459-5466.

[13] D. F. B. Haeufle, M. D. Taylor, S. Schmitt, and H. Geyer, "A clutched parallel elastic actuator concept: Towards energy efficient powered legs in prosthetics and robotics," in 2012 4th IEEE RAS \& EMBS International Conference on Biomedical Robotics and Biomechatronics (BioRob). Rome, Italy: IEEE, June 2012, pp. 1614-1619.

[14] W. Roozing, Z. Ren, and N. G. Tsagarakis, "An efficient leg with series-parallel and biarticular compliant actuation: Design optimization, modeling, and control of the eLeg," The International Journal of Robotics Research, vol. 40, no. 1, pp. 37-54, Jan. 2021

[15] X. Liu, A. Rossi, and I. Poulakakis, "A Switchable Parallel Elastic Actuator and its Application to Leg Design for Running Robots," IEEE/ASME Transactions on Mechatronics, vol. 23, no. 6, pp. 2681-2692, Dec. 2018.

[16] B. L. Proffen, M. McElfresh, B. C. Fleming, and M. M. Murray, "A comparative anatomical study of the human knee and six animal species," The Knee, vol. 19, no. 4, pp. 493-499, Aug. 2012.

[17] M. Hutter, et al., "ANYmal - toward legged robots for harsh environments," Advanced Robotics, vol. 31, no. 17, pp. 918-931, 2017.

[18] Y.-H. Chang and L. H. Ting, "Mechanical evidence that flamingos can support their body on one leg with little active muscular force," Biology Letters, vol. 13, no. 5, p. 20160948, May 2017.

[19] F. Grimminger, et al., "An Open Torque-Controlled Modular Robot Architecture for Legged Locomotion Research," IEEE Robotics and Automation Letters, vol. 5, no. 2, pp. 3650-3657, Apr. 2020.

[20] J. Pratt, C.-M. Chew, A. Torres, P. Dilworth, and G. Pratt, "Virtual Model Control: An Intuitive Approach for Bipedal Locomotion," The International Journal of Robotics Research, vol. 20, no. 2, pp. 129-143, Feb. 2001.

[21] O. Khatib, "A unified approach for motion and force control of robot manipulators: The operational space formulation," IEEE Journal on Robotics and Automation, vol. 3, no. 1, pp. 43-53, Feb. 1987.

[22] J. Hwangbo, V. Tsounis, H. Kolvenbach, and M. Hutter, "Cable-driven actuation for highly dynamic robotic systems," in 2018 IEEE/RSJ International Conference on Intelligent Robots and Systems (IROS). IEEE, 2018, pp. 8543-8550. 